\title{
P53 and thymidine dimer induction in daily low emission broad band uv treatment
}

\begin{abstract}
Background/purpose: Phototherapy is widely used as treatment modality for psoriasis. Recently we reported that daily low-dose phototherapy can be effective as treatment for psoriasis patients. To study the carcinogenic effects in patients receiving this treatment, nineteen patients underwent skin biopsies.
\end{abstract}

Methods: P53 and thymidine dimers were assessed in skin biopsies of psoriasis patients (8 controls, 7 patients with daily low emission broad band UV- treatment, 4 conventional NB-UVB-treatment).

Results: Staining for the presence of p53 in epidermal cells was unremarkable and not significantly different between daily low-dose UV treatment $(1 / 7=14.3 \%$ p53 positive $)$ and treatment without phototherapy $(2 / 8=25.0 \% \mathrm{p} 53$ positive $)(\mathrm{p}=1.0)$. Thymidine dimers were investigated by FACS analysis. No difference was found in the amount of fluorescence of the cells between daily low-dose UV-treatment and no phototherapy groups $(p=0.189)$ or in the percentage of dimers found $(p=0.463)$. A significant $(p=0.006)$ difference in amount of thymidine dimers was found in patients undergoing conventional TL01 phototherapy compared to the daily low-emission UV-treated group as well as compared to patients not undergoing phototherapy.

Conclusion: These results suggest that treatment of psoriasis patients with daily low-dose UV result in a low carcinogenic risk. Further studies however are needed to confirm these results.

Keywords: p53, thymidine dimes, psoriasis, phototherapy, low-emission
Volume 4 Issue 2 - 2016

\author{
Franken SM,' Spiekstra SW,' Witte BI, ${ }^{2}$ Pavel \\ $\mathrm{S},{ }^{3}$ Rustemeyer $\mathrm{T}^{\prime}$ \\ 'Department of Dermatology, VU University Medical Center, \\ Netherlands \\ ${ }^{2}$ Department of Epidemiology and Biostatistics, VU University \\ Medical Center, Netherlands \\ ${ }^{3}$ Department of Dermatology, Mauritsklinieken, Netherlands
}

Correspondence: Franken SM , Department of Dermatology, VU University Medical Center, De Boelelaan II 18, 108IHV Amsterdam, Netherlands, , Fax 31 (0)204440I48,

Emails.franken@vumc.nl

Received: October 13, 2016 | Published: October 21, 2016
Abbreviations: BB-UVB, broad band UVB; NB-UVB, narrow band UVB; ROS, reactive oxygen species

\section{Introduction}

Phototherapy is a widely used treatment for multiple skin diseases, but particularly in psoriasis. ${ }^{1,2}$ Many modalities for phototherapy have been used and investigated in the treatment of psoriasis patients: UVA with psoralens (so called local or systemic PUVA), broadband UVB (BB-UVB) and narrowband UVB (NB-UVB). These UV-treatments can be given in different schemes - daily, twice or thrice weekly- and they can be used even in combination with systemic therapy, for instance to enhance the effect of retinoids, or they can be combined with local treatment (e.g. the Goeckerman regimen). ${ }^{1,3,4}$

While phototherapy is often a very effective treatment option in psoriasis patients ${ }^{1}$, there are risks involved. ${ }^{5-10}$ The well-known acute risks of phototherapy are sunburn and immunosuppression, however many physicians especially worry about a potential carcinogenic risk of this treatment.

\section{Carcinogenic risks}

Multiple studies have proven a relatively high carcinogenicity of PUVA-treatment. ${ }^{5,8-10}$ This is not surprising since the phototoxic psoralens bind covalently to DNA (so called DNA intercalation) where they function as a source of reactive oxygen species during the UVA exposure. Especially squamous cell carcinomas and their potential precursors, actinic keratoses, have been reported after longterm PUVA treatments.

Several studies have looked into the incidence of skin cancer in patients undergoing Goeckerman regimen (crude coal tar combined with UV-radiation) without being able to ascertain an increased risk of skin malignancies. ${ }^{11,12}$

NB-UVB and BB-UVB seem to be relatively safe. ${ }^{13}$ No association was found in a study by Hearn et al. ${ }^{6}$ which focussed on the UVinduced skin cancer in patients undergoing NB-UVB phototherapy. However, the authors warn that further studies are required to assess the risk, especially if this might be a dose-dependent or cumulative risk, as is the case in PUVA-treatment.

Carcinogenicity is mainly based on the direct genotoxic effects of UV-radiation, and to a lesser extent on the generation of reactive oxygen species (ROS). The latter can directly cause activation of cytochrome $\mathrm{c}$ in mitochondria, resulting in apoptosis. The phototoxic effects of UV-radiation on the DNA of cells are the main cause of apoptosis and cellular damage. They are the most investigated mechanism in this context.

UVB-radiation is absorbed by cellular DNA strands which can lead to characteristic $\mathrm{C}$ to $\mathrm{T}$ transitions. These transitions and thymidine dimers are usually repaired, but after an extensive and frequent photodamage, the cells do not have enough time for reparation and some repair errors can be made. A specific UV-signature in the generation of thymidine dimers has been identified in squamous cell carcinomas. This suggests an etiological role of UV-radiation in the development of these skin tumours. ${ }^{8-10,14,15}$

Another factor that takes important part in UV-carcinogenesis is protein $\mathrm{p} 53$. This is a tumor suppressor gene that plays an essential role in cell cycle control. It can route the cell to apoptosis, repair or cell differentiation. ${ }^{11,16}$ It is induced to direct the cell to one of the mentioned pathways when cellular damage by UV-radiation occurred. 
Its induction and mutations have been found in several malignant diseases (squamous cell carcinomas, basal cell carcinomas, etc.), precancerous diseases (Bowens disease, actinic keratosis) and benign diseases including psoriasis. ${ }^{17,18}$ It has been argued that p53 plays a possible role in the pathogenesis of psoriasis. ${ }^{16,18}$

Several studies have shown a significant rise in staining of p53 in psoriatic skin versus normal skin. In non-lesional psoriatic skin, a trend seems to be present in $\mathrm{p} 53$ induction, but in two different studies no significant difference could be found. ${ }^{19,20}$ However, a significant induction of $\mathrm{p} 53$ by UV-exposure was seen. ${ }^{19}$

After UV-radiation an induction of p53 can be detected in 12-24 hours, although a slow rise is already distinguishable after 4 hours. ${ }^{21,22}$

\section{Treatment with daily low emission UV}

Recently, we performed a study with psoriasis patients treated with a daily low dose UV (1 standard erythemal dose (SED) only). In this clinical investigation, a significant drop in PASI-scores was found. This was accomplished in 6 months follow-up. This clinical effect was accompanied by a significant rise in vitamin D $25(\mathrm{OH})$ D blood concentration. ${ }^{23}$ A long-term use phototherapy, albeit with a low UV-emission, however, always rises the question of potential carcinogenic effects. As the main goal of the above mentioned study was to ascertain the efficacy of this therapy for daily use, it is therefore of special importance to evaluate the safety profile of such treatment regimen.

\section{Materials \& methods}

Study design was approved by the Medical Ethical Committee of the VU University Medical Center (VUmc) and was performed in accordance to the declaration of Helsinki (2008). The results were gathered in connection with a larger clinical effectiveness study described elsewhere. ${ }^{32}$

Patients were randomised in two groups: one group undergoing daily low emission broad band (BB) UV-treatment (Dermasun Helios, Dermasun Medical BV, The Netherlands) and using mometason ointment once daily if they deemed this necessary. The second group only used mometason ointment once daily if necessary. Randomisation took place by choosing a non-marked envelop by patients themselves with a note allocating them to the particular treatment group.

All patients were asked to refrain from sunbathing, use of tanning booth or sunny holidays. Patients did not use oral vitamin D supplements, systemic immunosuppressive medication and did not have a skin malignancy in their medical history.

To measure the DNA damage caused by the daily low emission phototherapy UV-treatment, ten randomly selected patients from each group were asked to allow two skin biopsies of $4 \mathrm{~mm}$ after at least 2 months of ongoing treatment. These biopsies were used to measure the presence of p53-mutations and thymidine dimers. Fifteen patients gave their consent, eight patients from the control group and seven patients from the home-treatment group.

As a positive control reference, four psoriasis patients undergoing conventional NB-UVB treatment (during at least two months) given thrice a week underwent skin biopsies as well. All biopsies were taken directly after UV-treatment.

P53-staining was performed routinely at the Pathology department of the VU medical center with monoclonal mouse anti-human p53 protein (Dako, Glostrup, DK) staining both p53 and mutant p53. Sections of adenocarcinoma of the colon were used as $\mathrm{p} 53$ positive controls.
P53 induction was visually assessed on two separate occasions in a blinded manner. Histological sections were assessed for an estimated percentage of positive cells and their intensity. A biopsy was deemed positive if $80 \%$ of keratinocytes was stained by p53 immunohistochemistry and $30 \%$ of those cells had a high-intensity staining. Examples of a p53 positive and negative staining are shown in Figure 1.

Thymidine dimers were measured essentially as described in the protocol of Rouget et al., ${ }^{24}$ In brief: Biopsies were incubated with $0.1 \%$ trypsin solution (Gibco, Paysly, UK) at $37^{\circ} \mathrm{C}$ for 10 minutes and shacked vigorously. After 5 minutes $400 \mathrm{G}$ centrifugation at $4^{\circ} \mathrm{C}$ the supernatant was discarded and the cells were resuspended with a 50 mM EDTA (Sigma-Aldrich, St., Louis, MO, USA) in PBS solution and fixed by adding 3 volumes of $100 \%$ ethanol at $-20^{\circ} \mathrm{C}$ and centrifuged as described above. Cells were incubated for 20 minutes at room temperature with a solution of $0.5 \%$ Triton-X100 (SigmaAldrich) in $2 \mathrm{~N} \mathrm{HCl}$ solution. Cells were then washed with a $0.1 \mathrm{M}$ $\mathrm{Na} 2 \mathrm{~B} 4 \mathrm{O} 7$ (Sigma-Aldrich) and incubated by a $100 \mathrm{ug} / \mathrm{ml}$ RNASE A solution (In Vitrogen, Carlsbad, CO, USA) at $37^{\circ} \mathrm{C}$ for 60 minutes. After washing with FACS-buffer (PBS, $0.1 \%$ azide, $0.1 \%$ BSA) antithymidine dimer antibodies (1:50) (Kamiya Biomedical company, Seattle, WA, USA) were incubated at room temperature for 1 hour. After washing, rabbit anti-mouse IgG-Fitcconjungated antibodies (1:10) (DAKOcytomation, Glostrup, DK) were added and incubated for at $4^{\circ} \mathrm{C}$ in the dark for 30 minutes.

As an internal control, $4 \mathrm{~mm}$ biopsies of donated foreskin were irradiated with $2.2 \mathrm{~J} / \mathrm{cm} 2$ at $320 \mathrm{~nm}$ (Spectral Luminator, Oriel instruments). Unexposed foreskin was measured in every run as well. Samples treated with only the secondary antibodies were used as a negative control. Samples were measured and analysed using the BD Facscalibur (BD, San Jose, CA, USA). Typical results from control foreskin biopsies and UV radiated foreskin are shown in Figure 2.
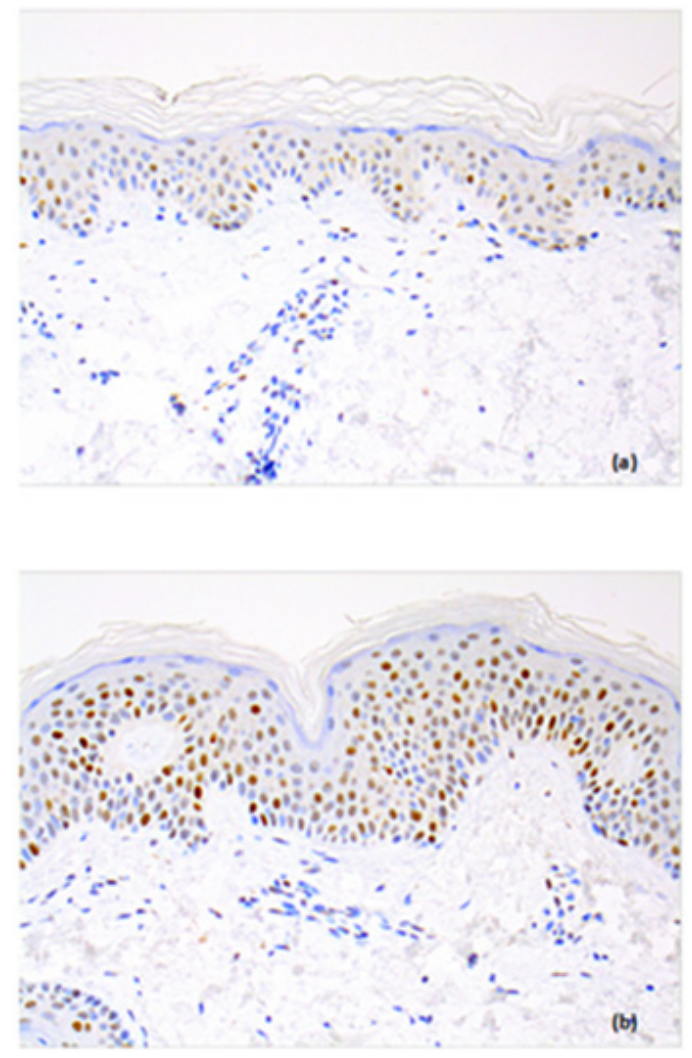

Figure I Example of negative (a) and positive (b) p53-staining in skin biopsy. 

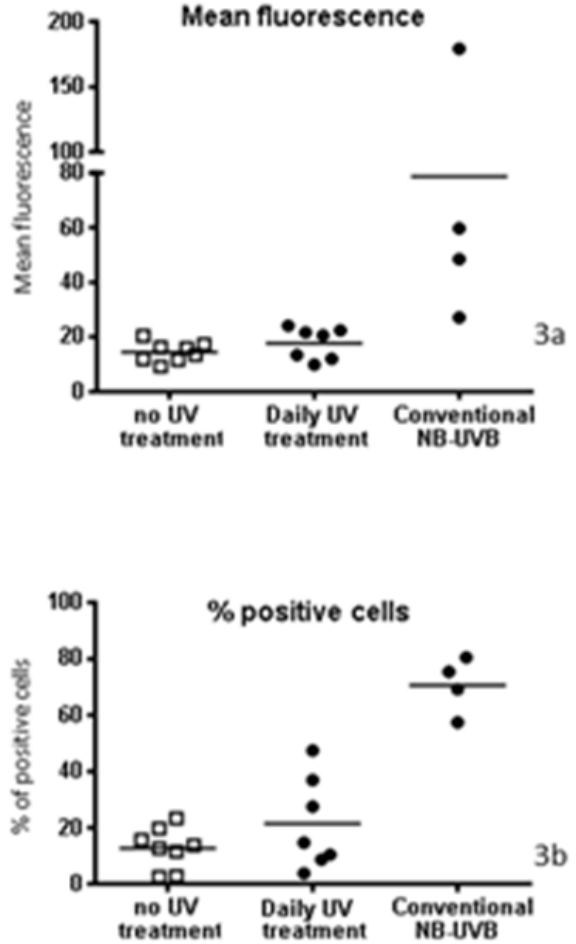

Figure 2 Scatter plot of a representative skin isolate and histogram plot of a typical non UV-exposed- (grey area) and, UV-B exposed foreskin (White area) are shown. Showing a clear increase of fluorescence intensity, showing an increase of CPD-dimers after UV-B exposure of the skin biopsies.

Percentage of positive cells was determined by using unexposed foreskin biopies as negative control. For each experimental run a UV-B exposed foreskin was used as an internal positive control for reproducibility of the assay.

\section{Statistics}

Statistical analyses were performed using SPSS (version 20, IBM Corp., Armonk, NY). A p-value $<0.05$ was considered to indicate statistical significance, a p-value $<0.1$ a statistical trend. Outcomes are described by either frequencies and percentages or by median and range. Differences between continuous variables (mean fluorescence and percentage of positive cells) were analysed by the Mann-Withney U-test, while differences in $\mathrm{p} 53$-staining were analysed by the Fisher's exact test.

\section{Results}

In nineteen patients ( 8 controls without phototherapy, 4 patients with conventional NB-UVB treatment, 7 patients with daily low emission BB UV- treatment) two $4 \mathrm{~mm}$ skin biopsies were taken from an area of the body that would normally not be sun exposed during the winter (buttocks, groin and extensor side of the upper arm). Patients undergoing phototherapy were asked to present themselves for the biopsy directly after irradiation. Baseline characteristics of patients can be found in Table 1.

Staining for the presence of p53 in epidermal cells was not significantly different between daily low-dose UV treatment $(1 / 7=14.3 \%$ p53 positive $)$ and treatment without phototherapy $(2 / 8=25.0 \%$ p53 positive $)(p=1.0$, Table 2$)$.

P53 upregulation has been described in prior literature. ${ }^{19,25,26}$ P53- staining was therefore not assessed in patient undergoing conventional NB-UVB treatment to minimize the amount of biopsies taken.

Thymidine dimers were investigated by FACS analysis. Although a small up-regulation was seen in the amount of fluorescence of the cells and in the percentage of positive cells in patients undergoing daily low emission BB UV vs no phototherapy, this trend was not significant (Firure 3a \& 3b). No difference was found in the amount of fluorescence of the cells between the daily low dose BB UVtreatment and no phototherapy groups $(\mathrm{p}=0.19)$ or in the percentage of thymidine dimers found $(\mathrm{p}=0.46)$.
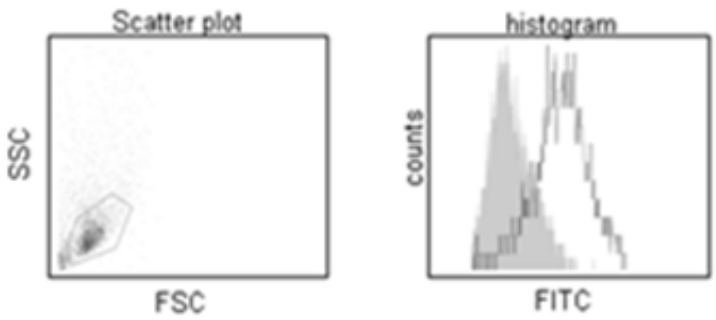

Figure 3a\&b Mean fluorescence of the fluorescence signal of bound antithymidine dimers antibodies in biopsy derived cells from patients without UV treatment, daily low emission broad band UV treatment and conventional NB-UVB, b: Percentage of dimer positive cells in skin biopsies of patients undergoing daily low emission broad band UV treatment and conventional NB-UVB.

To confirm that our method was effective and to clarify the difference with conventional phototherapy, 4 patients undergoing conventional NB-UVB treatment for at least two months were asked to allow a skin biopsy. A significant $(p=0.006)$ difference was found in these patients compared to the daily low-emission BB UV-treated group as well as compared to patients not undergoing phototherapy.

\section{Discussion}

Although the groups in this study were small, the results of our measurements show clear differences. Only a small elevation of thymidine dimers was found in the group receiving daily low emission BB UV treatment compared to patient without any phototherapy. This elevation was, however statistically non-significant. A slight elevation would be expected as patients were receiving any form of phototherapy: a slight UV-damage is a logical result of an effective treatment. However, in comparison with the controls treated with conventional narrowband phototherapy however, the rise in thymidine dimers was remarkably lower. The difference between these groups was statistically significant.

P53-staining was also non-significantly different between patients using mometason only (without UV-therapy) and with daily lowemission UV-therapy. Interestingly, in patients using mometason ointment only the baseline p53 staining was higher than expected. This is in line with prior reports in literature in which a higher p53activity may be found in psoriatic lesions. Although biopsies were not taken from lesional skin, non-lesional skin seems to have a higher baseline p53 staining as well. ${ }^{19,20}$

In light of the results concerning the thymidine dimers, a slight induction of p53 in the skin biopsies in patients undergoing daily lowdose-UV treatment was expected. Remarkably, this induction was not found, p53-activity was equal in both groups. The evaluation method for $\mathrm{p} 53$ staining was semiquantative: during two separate occasions, a visual estimation on positive staining of p53 and its intensity was done. On one of these occasions, a pathologist took part in the appraisal. 
Table I Baseline characteristics of included patients

\begin{tabular}{llll}
\hline & No Phototherapy $(\mathbf{n = 8})$ & Conventional NB-UVB (n=4) & Daily BB-UV (n=7) \\
\hline Male, $\mathrm{n}(\%)$ & $5(62.5 \%)$ & $3(75.0 \%)$ & $4(57.1 \%)$ \\
Age, Mean \pm SD & $4 I .5 \pm 9.8$ & $48.8 \pm 13.7$ & $43.3 \pm .15 .1$ \\
\hline Fitzpatrick Skin Type & & & $1(14.3 \%)$ \\
\hline Type I, n (\%) & $0(0 \%)$ & $0(0 \%)$ & $6(85.7 \%)$ \\
Type 2, n (\%) & $7(87.5 \%)$ & $2(50.0 \%)$ & $0(0 \%)$ \\
Type 3, n (\%) & I (I2.5\%) & $2(50.0 \%)$ & 4 months \\
Treatment time to biopsy, mean & N/A & 3 months & \\
\hline
\end{tabular}

Table 2 Descriptive statistics of p53-mutations and thymidine dimers

\begin{tabular}{llll}
\hline & No Phototherapy $(\mathbf{n}=8)$ & Conventional NB-UVB (n=4) & Daily BB UV (n=7) \\
\hline p53 positive, $n(\%)$ & $2(25.0 \%)$ & Not tested & I (I4.3\%) \\
Fluorescence, median [range] & I4.7 [9.2 -20.4] & $54.1[27.1-179.2]$ & $20.6[9.9-24.2]$ \\
Percentage dimers, median [range] & $13.2[2.5-23.3]$ & $72.3[57.4-80.5]$ & $14.7[3.8-47.5]$ \\
\hline
\end{tabular}

Biopsies taken in the UV-poor winter season from UV-protected skin (buttocks or thighs) in control patients, however showed a relatively high baseline induction of $\mathrm{p} 53$. This is in line with previous literature. ${ }^{19,20}$

The lack of p53 induction during daily low emission phototherapy has several possible explanations. The induction, if present, should have been demonstrated, as patients undergo daily phototherapy. The peak in p53 induction is after 12-24 hours, but a rise is visible in less than 4 hours. ${ }^{22}$ The results therefore could be suggestive to exemplify that low emission broadband phototherapy in a daily regimen can be regarded as a safe treatment. A small, non-significant rise in thymidine dimers (CPD's) was found, suggesting that minimal damage is present and confirms a biologic effect of the low-emissions devices. It might be possible that this damage is so minimal that the NER-pathway is able to rapidly repair the damage. P53 is therefore not induced or so minimally that gross visual detection by an experienced pathologist is not possible.

The clear, gradual elevation of vitamin D $(25(\mathrm{OH}) \mathrm{D} 3)$ over time shows that the daily low-dose UV exposure suffices to induce elevation of vitamin D levels. It therefore seems possible to use UV radiation in a none or minimally damaging fashion, something that has not been studied and reported in prior publications. So far, the assumption has been that treatment with phototherapy, although with high suberythematous dosages, obligatorily meant that the production of CPD and $\mathrm{p} 53$-induction would follow.

Because the tested groups were small, further investigation to confirm this observation and strengthen these conclusions is needed. As carcinogenic risk is often a cumulative one, it is important to regularly check patients undergoing this treatment modality for development of skin malignancies. Prior studies ${ }^{28,29}$ have demonstrated that in particular p53 mutation clones proliferate while exposed to phototherapy, and diminish after UV exposure.

These findings emphasize the potential carcinogenic risk of longterm UV-treatment regimes. However, in this study using a low-dose BB UV-emitting device no significant p53 changes have been found. This suggests a low risk of such unwanted side effects from this device and treatment regime. In light of potential long-term effects of daily low-dose BB UV-radiation, future research should also focus on immunological effects after years. For the time being, a conservative approach seems wise to regularly perform a total skin inspection in patients using this treatment modality in a prolonged fashion to check for possible skin malignancies as it is recommended for all other UVtreatments.

\section{Conflicts of interest}

None.

\section{Funding}

None.

\section{References}

1. Chen X, Yang M, Cheng Y, et al. Narrow-band ultraviolet B phototherapy versus broad-band ultraviolet B or psoralen-ultraviolet A photochemotherapy for psoriasis. Cochrane Database Syst Rev. 2013;10:CD009481.

2. Racz E, Prens EP. Phototherapy and photochemotherapy for psoriasis. Dermatol Clin. 2015; 33(1):79-89.

3. Baros DN, Gajanin VS, Gajanin RB, et al. Comparative analysis of success of psoriasis treatment with standard therapeutic modalities and balneotherapy. Med Pregl. 2014;67:154-160.

4. MenterA, Cram DL. The Goeckerman regimen in two psoriasis day care centers. J Am Acad Dermatol. 1983;9(1):59-65.

5. Archier E, Devaux S, Castela E, et al. Carcinogenic risks of psoralen UV-A therapy and narrowband UV-B therapy in chronic plaque psoriasis: a systematic literature review. J Eur Acad Dermatol Venereol. 2012;26(Suppl 3):22-31.

6. Hearn RM, Kerr AC, Rahim KF, et al. Incidence of skin cancers in 3867 patients treated with narrow-band ultraviolet B phototherapy Br. $J$ Dermatol. 2008;159(4);931-935.

7. Kunisada M, Kumimoto H, Ishizaki K, et al. Narrow-band UVB induces more carcinogenic skin tumors than broad-band UVB through the formation of cyclobutane pyrimidine dimer. $J$ Invest Dermatol. 2007;127(12):2865-2871.

8. Stern RS. The risk of squamous cell and basal cell cancer associated with psoralen and ultraviolet A therapy: a 30-year prospective study. $J$ Am Acad Dermatol. 2012;66(4):553-562.

9. Stern RS, Bolshakov S, Nataraj AJ, et al. p53 mutation in nonmelanoma skin cancers occurring in psoralen ultraviolet a-treated patients: evidence for heterogeneity and field cancerization. $J$ Invest Dermatol. 2002;119(2):522-526.

10. Stern RS, Liebman EJ, Väkevä L. Oral psoralen and ultraviolet-A light (PUVA) treatment of psoriasis and persistent risk of nonmelanoma skin cancer. PUVA Follow-up Study. J Natl Cancer Inst. 1998;90(17):1278-1284

11. Hannuksela-Svahn A, Pukkala E, Läärä E, et al. Psoriasis, its treatment, and cancer in a cohort of Finnish patients. $J$ Invest Dermatol. 2000;114(3):587-590. 
12. Hannuksela-Svahn A, Pääkkö P, Autio P, et al. Expression of p53 protein before and after PUVA treatment in psoriasis. Acta Derm Venereol. 1999;79(3):195-199.

13. Larkö O, Swanbeck G. Is UVB treatment of psoriasis safe? A study of extensively UVB-treated psoriasis patients compared with a matched control group. Acta Derm Venereol.1982;62(6):507-512.

14. Mitchell DL. The induction and repair of lesions produced by the photolysis of (6-4) photoproducts in normal and UV-hypersensitive human cells. Mutat Res. 1988;194(3):227-237.

15. Mitchell DL. The relative cytotoxicity of (6-4) photoproducts and cyclobutane dimers in mammalian cells. Photochem Photobiol. 1988;48(1):51-57.

16. Peritz AE, Gasparro FP. Psoriasis, PUVA, and skin cancer--molecular epidemiology: the curious question of T-->A transversions. J Investig Dermatol Symp Proc. 1999;4(1):11-16.

17. Soini Y, Kamel D, Pääkkö P, et al. Aberrant accumulation of p53 associates with $\mathrm{Ki} 67$ and mitotic count in benign skin lesions. $\mathrm{Br} J$ Dermatol. 1994;131(4):514-520.

18. Tadini G, Cerri A, Crosti L, et al. P53 and oncogenes expression in psoriasis. Acta Derm Venereol Suppl (Stockh). 1989;146:33-35.

19. Yazici AC, Karabulut AA, Ozen O, et al. Expression of p53 in lesions and unaffected skin of patients with plaque-type and guttate psoriasis: a quantitative comparative study. J Dermatol. 2007;34(6):367-374.

20. Baran W, Szepietowski JC, Szybejko-Machaj G. Expression of p53 protein in psoriasis, Acta Dermatovenerol. Alp Panonica. Adriat. 2005;14(3):79-83

21. de Winter S, Vink AA, Roza L, et al. Solar-simulated skin adaptation and its effect on subsequent UV-induced epidermal DNA damage. J Invest Dermatol. 2001;117:678-682.
22. Tjioe M, Smits T, van de Kerkhof PC, et al. The differential effect of broad band vs narrow band UVB with respect to photodamage and cutaneous inflammation. Exp Dermatol. 2003;12:729-733.

23. Franken SM, Witte B, Pavel S, et al. Psoriasis and daily low-emission phototherapy: effects on disease and vitamin D level. Photodermatol Photoimmunol Photomed. 2014;31(2):83-89.

24. Rouget R, Auclair Y, Loignon M, et al. A sensitive flow cytometry-based nucleotide excision repair assay unexpectedly reveals that mitogenactivated protein kinase signaling does not regulate the removal of UV-induced DNA damage in human cells. J Biol Chem. 2008;283(9): 5533-5541.

25. DeSilva B, McKenzie RC, Hunter JA, et al. M Local effects of TL01 phototherapy in psoriasis. Photodermatol Photoimmunol Photomed. 2008;24:268-269.

26. Jasim ZF, Lioe TF, McKenna KE, et al. The effect of ultra violet B (TL01) phototherapy on epidermal expression of p53 protein in psoriatic plaques. Photodermatol Photoimmunol Photomed. 2006;22(1):12-17.

27. Roshan A, Jones PH. Chronic low dose UV exposure and p53 mutation: tilting the odds in early epidermal preneoplasia? Int J Radiat Biol. 2002;88(10):682-687.

28. Zhang W, Remenyik E, Zelterman D, et al. Escaping the stem cell compartment: sustained UVB exposure allows p53-mutant keratinocytes to colonize adjacent epidermal proliferating units without incurring additional mutations. Proc Natl Acad Sci U S A. 2001;98(24):13948-13953

29. Pittelkow MR, Perry HO, Muller SA, et al. Skin cancer in patients with psoriasis treated with coal tar. A 25-year follow-up study. Arch Dermatol. 1981;117(8):465-468. 